\title{
Apertureless Near-field Probe Design with Correlation to Field Enhancement and Focal Delocalization
}

Derek B. Nowak(dbn@pdx.edu), Jeff J. Doughty, A. J. Lawrence, Erik J. Sánchez (esanchez@pdx.edu)

Department of Physics, Portland State University, P.O. Box 751, Portland, OR 97207

Traditional light microscopy is fundamentally limited in its spatial resolution due to diffraction. Ernest Abbe was the first to define the diffraction limit in terms of the numerical aperture (NA) of the focusing lens and the wavelength of the illuminating light used. In general, when a high NA objective lens is used, this limit is roughly half the wavelength of the illumination source. Confocal techniques have extended this limit to one-third the illumination wavelength, with minimal modification to the original microscope design. More complex techniques include "super-resolution" far-field imaging and near-field scanning probe methods. Both of these fields have demonstrated resolutions on the order of $10 \mathrm{~s}$ of nanometers but they are not without their limitations. "Superresolution" far-field methods rely on known dye-labels, complex laser systems and correlated timing of pulses and emission detection [1,2,3]. Near-field methods are confined to thin regions of samples that are less than an illumination wavelength in height, and can demonstrate quenching of the fluorescent response of dye molecules [4]. Advantages to the use of near-field methods are the ability to image without previous knowledge of the sample and, most importantly, near-field's reach into Raman spectroscopy. Raman's near-instantaneous response does not allow for simple time correlation of an emission response, a key requirement in "super-resolution" methods. It is these capabilities that continue to drive the discipline of near-field microscopy. In particular, the materials sciences benefit from the high resolutions obtainable with near-field microscopy. Nano-materials can be analyzed with Raman spectroscopy for molecular and atomic bond information or photoluminescence and fluorescence responses to determine bulk optical properties [5].

Near-field scanning optical microscopy (NSOM or SNOM) uses a combination of a shear force microscope (SFM) and an inverted optical microscope. Originally, NSOM was developed by using a glass pipette or optical fiber to propagate the excitation source to a confined aperture of tens of nanometers [6]. Later, it was determined that one could use solid metal tips, illuminated in the far field by the excitation wavelength of interest. This technique has been named tip enhanced near-field optical microscopy (TENOM) [7]. A benefit of TENOM is the improved topographic resolution and optical detection excitation efficiency. The tips are custom-designed using finite difference time domain (FDTD) modeling techniques, then fabricated with the use of a focused ion beam microscope. The quality of the tip design is determined by the field enhancement obtainable. The greater the field enhancement of the tip, the greater the ratio of near-field to far-field background contribution. Ultimately, the elimination of the far-field signal by a decrease of illumination (laser) power will provide the best signal-to-noise ratio in the near-field images.

Determining the correct geometries for the imaging probe is paramount. Combining this with the complication of design and operation of the near-field microscope itself can be daunting. To minimize the complications of probe testing, we have developed a microscope that employs twophoton, non-linear excitation to allow for the imaging of the fluorescence from almost any visible fluorophore at resolutions below $20 \mathrm{~nm}$ without changing filters or excitation wavelength. The microscope has the ability to image topographic, optical, and electronic state information for single- 
molecule identification, operating at atmospheric pressure, room temperature, and in solution. A single computer, simple control circuits, FPGA data acquisition, and a simplified optical system control the microscope $[8,9]$. This versatility enables the end user to custom-design experiments from confocal far-field single molecule imaging to high resolution scanning probe microscopy. We are currently working on the dissemination of the microscope as an open system design [10].

We have demonstrated probe designs that work well with epi-illumination from below [11]. These designs remove the polarization dependence on the illumination source, simplifying the alignment procedure for imaging [12]. FIG 1 shows a recent near-field image acquired with one of the probe designs. Currently, the designs are being optimized to maximize field enhancement, thereby lowering the background contributions from the excitation source. Furthermore, we have demonstrated that the probe design can withstand the increased laser power needed for two-photon excitation with a continuous wave laser [9]. A CW laser source can greatly reduce the instrument cost and simplify the laser requirements for imaging. Raman spectroscopy capabilities currently under development will also be discussed.
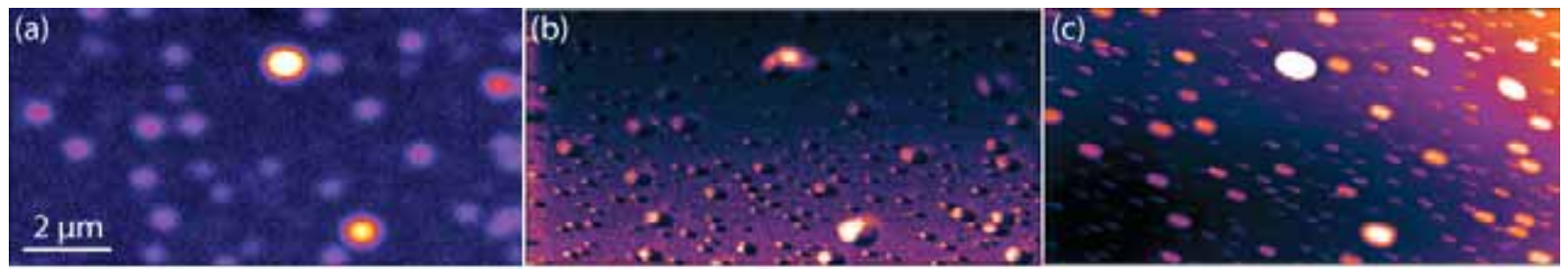

FIG 1: Two-photon imaging of Rhodamine $6 \mathrm{G}$ dye excited at $833 \mathrm{~nm}$ with a mode-locked laser operating at $52 \mathrm{fs}$ pulses. Air-drying a $\mu \mathrm{M}$ concentration of R6G in methanol forms the clusters of dye. (a) Far-field diffraction limited image with an average excitation power of $27.21 \mu \mathrm{W}$. (b) Near-field image with an average excitation power of $8.35 \mu \mathrm{W}$, same ROI as (a). The near-field image demonstrates the effect of quenching by the imaging probe. (c) Topographic scan, simultaneously acquired with (b). The imaging probe geometry is creating the artifacts seen in the topographic image. Close inspection between (a) and (b) shows a focal delocalization of roughly $600 \mathrm{~nm}$.

Work Funded by: NSF award DBI-0500812, with assistance from ECCS-NSF-0520891, DMR-NSFREU-\#0649280, and NSF-069280, ONAMI/ONR awards (\#N00014-07-1-0457), (\#N00014-08-11237), (\#N00014-10-1-0082), the Western Institute of Nanoelectronics (WIN) / NRI.

References:

[1] S. W. Hell, J. Wichmann, Optics Letters 19, 780-782 (1994).

[2] E. Betzig, et al., Science 313, 1642-1645 (2006).

[3] M. J. Rust, M. Bates, X. Zhuang, Nature Meth. 3, 793-796 (2006).

[4] J. T. Krug, E. J. Sánchez, X. S. Xie, Appl. Phys. Lett. 86, 233102, (2005).

[5] A. Hartschuh, et al., Phys. Rev. Lett. 90, 9, March 7, 2003, 095503-1.

[6] E. Betzig, et al., Science 251, 1468-1470 (1991).

[7] E. J. Sánchez, L. Novotny, X.S. Xie, Phys. Rev. Lett. 82, 20 1999, 4014.

[8] D. B. Nowak, et al., Unpublished manuscript.

[9] D. B. Nowak, A. J. Lawrence, E. J. Sánchez, Applied Optics 49, 35 6771, (2010).

[10] D. B. Nowak, et al., "ANSOM Project," http://ansom.research.pdx.edu/

[11] D. B. Nowak, Ph.D dissertation, Portland State University, UMI 3419910, (2010).

[12] J. J. Doughty, MS thesis, Portland State University, UMI 1482725, (2010). 